\title{
NOTE ON THE CONVERGENCE OF A SEQUENCE OF APPROXIMATING POLYNOMIALS*
}

\section{BY DUNHAM JACKSON}

Various results have been published, by the present writer and others, with regard to the convergence of sequences of approximating polynomials defined by minimizing an integral of the form

$$
\int_{a}^{b} \rho(x)\left|f(x)-P_{n}(x)\right|^{m} d x
$$

in which $f(x)$ is a function defined and continuous for $a \leqq x \leqq b$, and subject to such further hypotheses as the particular convergence proof in question demands, $\rho(x)$ is summable and nonnegative over the interval, $m$ is a given positive number $>1$, and $P_{n}(x)$ is a polynomial of the $n$th degree (this expression being understood throughout to mean a polynomial of the $n$th degree at most). The proof of convergence as $n$ becomes infinite is most easily obtained if $\rho(x)$ has a positive lower bound, at least over an interval containing the point at which convergence is to be shown. $\dagger$ The author has briefly discussed for the corresponding trigonometric case the question of convergence at a point at which the weight function vanishes, under certain restrictive hypotheses as to the manner of vanishing. $\ddagger$ The purpose of this note is to point out that if $f(x)$ is analytic in a sufficiently extended region of the complex plane the conditions relative to the vanishing of $\rho(x)$ can be greatly generalized. It is sufficient that $\rho(x)$ (supposed summable and non-negative over the interval of integration) be different from zero over a set of positive measure, or in other words that

$$
\int_{a}^{b} \rho(x) d x>0 .
$$

* Presented to the Society, September 9, 1930.

† See, for example, D. Jackson, The Theory of Approximation, New York, 1930, pp. 96-101.

$\ddagger A$ generalized problem in weighted approximation, Transactions of this Society, vol. 26 (1924), pp. 133-154; see pp. 153-154. See also the paper by Shohat in the Mathematische Annalen cited below. 
An existence proof for the approximating polynomial can be given without difficulty. Close restrictions on $f(x)$ are irrelevant for this purpose; it is sufficient that $f(x)$ be bounded and measurable. The exponent $m$ furthermore may be any positive number, not necessarily greater than unity. A discussion by the author for the case of trigonometric approximation* can be adapted immediately to prove the existence of a minimizing polynomial in the present problem, if $\rho(x)$ is bounded and measurable. It remains to show that the conclusion holds whenever $\rho(x)$ is summable. $\dagger$

The transition is almost immediate. Whether $\rho$ is bounded or not, the value of the integral (1) is a continuous function of the coefficients in $P_{n}(x)$. For bounded $\rho$, the existence proof consists essentially in showing that if an upper bound is assigned for the value of the integral, an upper bound is thereby imposed on the coefficients, so that the problem is solved by the theorem on the minimum of a continuous function in a closed region. If $\rho$ is unbounded, let $N$ be a positive number such that $0<\rho(x) \leqq N$ over a point set of positive measure; the fact that there is such an $N$ is assured by the hypotheses that have been laid down. Let a function $\rho_{N}(x)$ be defined as equal to $\rho(x)$ at points where $\rho \leqq N$, and let $\rho_{N}(x)=N$ where $\rho \geqq N$. If an upper bound is assigned for the integral (1), the corresponding integral with $\rho$ replaced by $\rho_{N}$ can not exceed this upper bound, since $\rho_{N} \leqq \rho$ everywhere, and application of the previous reasoning to the integral with the bounded function $\rho_{N}$ yields an upper bound for the coefficients in $P_{n}(x)$. The proof is then completed as before.

Under the assumption that $m>1$, the polynomial reducing the integral to its minimum is uniquely determined for each value of $n$. This may be deduced, as in similar situations elsewhere, from the observation that if two different polynomials give the integral the same value their average gives it a smaller one.

* Transactions of this Society, loc. cit., pp. 133-139.

$\dagger$ A somewhat different proof for the case $m \geqq 1$ is given by J. Shohat, On the polynomial and trigonometric approximation of measurable bounded functions on a finite interval, Mathematische Annalen, vol. 102 (1929), pp. 157175 ; pp. 160-161. See also J. Shohat, On the polynomial of the best approximation to a given continuous function, this Bulletin, vol. 31 (1925), pp. 509-514. 
Let it be supposed now that $f(x)$ is continuous for $a \leqq x \leqq b$. (This hypothesis will presently be replaced by a still more restrictive one.) For any specified $m>1$, and for each value of $n$, let $P_{n}(x)$ be the particular polynomial of the $n$th degree which minimizes the integral (1). As a general notation, let the symbol $[y]_{m-1}$ represent the value 0 if $y=0$, and the quantity $|y|^{m} / y$ if $y \neq 0$, so that $[y]_{m-1}$ has the absolute value of $|y|^{m-1}$ and the algebraic sign of $y$ itself. Then, if $Q_{n}(x)$ is any polynomial of the $n$th degree,

$$
\int_{a}^{b} \rho(x)\left[f(x)-P_{n}(x)\right]_{m-1} Q_{n}(x) d x=0 .
$$

The proof is an immediate generalization of one given by the writer elsewhere.* The generalization consists in the admission of the weight function $\rho(x)$, which was not present in the earlier discussion cited. Its presence, however, makes no difference in the argument, if it is noted that the elementary sufficient condition for differentiating under the sign of integration can be replaced by the assertion that

$$
\frac{d}{d y} \int_{a}^{b} \rho(x) \phi(x, y) d x=\int_{a}^{b} \rho(x) \phi_{y}(x, y) d x
$$

provided that $\phi$ and $\phi_{y}$ are continuous functions of both variables and $\rho(x)$ is summable.

This being granted, the reasoning proceeds as follows. If $f(x)-P_{n}(x)$ does not change sign at least $n+1$ times in $(a, b)$, a polynomial $Q_{n}(x)$ of the $n$th degree can be constructed so as to have the same sign as $f(x)-P_{n}(x)$ at all points where $f(x)-P_{n}(x) \neq 0$. The integrand in (2) then is positive or zero over the whole interval, and positive at all points where $\rho(x)$ and $f(x)-P_{n}(x)$ are both different from zero. Since the value of the integral must be zero, the integrand must vanish almost everywhere, and $f(x)-P_{n}(x)$ consequently must vanish wherever $\rho(x) \neq 0$, with the exception of a set of zero measure at most. As $\rho(x)$ by hypothesis is positive over a set of positive measure, $f(x)-P_{n}(x)$ must vanish throughout a set of positive measure, or else change sign at least $n+1$ times in the interval.

* On functions of closest approximation, Transactions of this Society, vol. 22 (1921), pp. 117-128; pp. 122-124. 
Let it be assumed finally that $f(x)$ is analytic for $a \leqq x \leqq b$, and analytic furthermore throughout a circle of the complex plane having its center at the middle point of the interval, and a radius greater than three times the half-length of the interval. If $P_{n}(x)$ is equal to $f(x)$ at all points of a set of positive measure, it is equal to $f(x)$ identically; in other words, $f(x)$ is itself a polynomial of the $n$th degree, and is its own polynomial of best approximation, of the $n$th or any higher degree. In the contrary case, $P_{n}(x)$ is equal to $f(x)$ for at least $n+1$ distinct values of $x$ in $(a, b)$. This condition being fulfilled for each value of $n$, a well known argument* shows that $P_{n}(x)$ converges uniformly toward $f(x)$ throughout a circle of the complex plane containing the interval $(a, b)$ in its interior. $\dagger$ The hypothesis that $f(x)$ is analytic throughout a smaller circle gives convergence in a correspondingly more restricted region.

The method is applicable also if the approximating polynomial is subjected to auxiliary conditions, of the sort discussed by the author in a previous paper, $\ddagger$ the weight function retaining the degree of generality admitted here.

The University of Minnesota

* See, for example, D. Jackson, On the approximate representation of analytic functions, this Bulletin, vol. 34 (1928), pp. 56-62, and references there to Hermite and Runge.

$\dagger$ By a slight modification, the convergence proof can be made to apply for $0<m \leqq 1$ also. For in any case (the function $f(x)$ being analytic) the multiplicities of the roots of the remainder $f(x)-P_{n}(x)$ on $(a, b)$ must add up to at least $n+1$, if $P_{n}(x)$ is any polynomial reducing the integral (1) to its minimum value, since otherwise a polynomial $Q_{n}(x)$, of the $n$th degree at most, could be constructed so as to have roots at the same points with the same multiplicities, and the same sign as the remainder where the remainder is different from zero; and then $\left[f(x)-P_{n}(x)\right] / Q_{n}(x)$, defined by continuity at the points where numerator and denominator vanish, would be positive over the whole interval and have a positive minimum $h$ there; and the subtraction of $h Q_{n}(x)$ would diminish the magnitude of the remainder at all points where it does not vanish, and would diminish the value of the integral. The count of multiplicities being sufficient for the proof of convergence, the conclusion is obtained as before. (Note added October 17, 1930.)

$\ddagger$ This Bulletin, loc. cit. 